\title{
A Remarkable Property of Concircular Vector Fields on a Riemannian Manifold
}

\author{
Ibrahim Al-Dayel ${ }^{1}$ (D), Sharief Deshmukh ${ }^{2}$ (D) and Olga Belova ${ }^{3, *(\mathbb{D})}$ \\ 1 Department of Mathematics and Statistics, College of Science, Imam Mohammad Ibn Saud Islamic University, \\ P.O. Box-65892, Riyadh 11566, Saudi Arabia; iaaldayel@imamu.edu.sa \\ 2 Department of Mathematics, College of Science, King Saud University, P.O. Box-2455, \\ Riyadh 11451, Saudi Arabia; shariefd@ksu.edu.sa \\ 3 Institute of Physical and Mathematical Sciences and IT, Immanuel Kant Baltic Federal University, \\ A. Nevsky str. 14, 236016 Kaliningrad, Russia \\ * Correspondence: olgaobelova@mail.ru; Tel.: +7-921-610-5949
}

Received: 6 March 2020; Accepted: 20 March 2020; Published: 29 March 2020

check for updates

\begin{abstract}
In this paper, we show that, given a non-trivial concircular vector field $u$ on a Riemannian manifold $(M, g)$ with potential function $f$, there exists a unique smooth function $\rho$ on $M$ that connects $u$ to the gradient of potential function $\nabla f$. We call the connecting function of the concircular vector field $u$. This connecting function is shown to be a main ingredient in obtaining characterizations of $n$-sphere $\mathbf{S}^{n}(c)$ and the Euclidean space $\mathbf{E}^{n}$. We also show that the connecting function influences on a topology of the Riemannian manifold.
\end{abstract}

Keywords: concircular vector field; connecting function; Ricci curvature; isometric to sphere; isometric to Euclidean space

\section{Introduction}

One of the important topics in differential geometry of a Riemannian manifold $(M, g)$ is the study of the influence of special vector fields on its geometry as well as topology. These special vector fields are geodesic vector fields, Killing vector fields, concircular vector fields, Jacobi-type vector fields, and conformal vector fields on a Riemannian manifold. Moreover, it is well known that their existence has considerable impact on the geometry of the Riemannian manifold and these vector fields are used in finding characterizations of spheres as well as Euclidean spaces (cf. [1-16]). Shandra studied some special types of concircular fields having no analogs for pseudo-Riemannian manifolds [17]. In [11], Fialkow initiated the study of concircular vector fields on a Riemannian manifold. A smooth vector field $u$ on a Riemannian manifold $(M, g)$ is said to be a concircular vector field if

$$
\nabla_{X} \boldsymbol{u}=f X, \quad X \in \mathfrak{X}(M),
$$

where $\nabla$ is the Riemannian connection on a Riemannian manifold $(M, g)$ and $f: M \rightarrow R$ is a smooth function and $\mathfrak{X}(M)$ is the Lie algebra of smooth vector fields on $M$ (see [18]). The smooth function appearing in the definition of the concircular vector field $u$ is called the potential function of the concircular vector field $u$. A concircular vector field $u$ is said to be a non-trivial concircular vector field if the potential function $f \neq 0$. In ([1], Theorem 3.1), Chen proved that, if $M$ is a Riemannian $n$-manifold which admits a nowhere zero concircular vector field, then $M$ is locally a warped product $I \times_{\varphi(s)} F$, where $\varphi(s)$ is a nowhere vanishing function and $F$ is a Riemannian $(n-1)$-manifold.

Note that a concircular vector field is a closed conformal vector field; a natural question arises: What is so special about a concircular vector field among closed conformal vector fields? In this paper, we answer this question by showing that to each non-trivial concircular vector $\boldsymbol{u}$ with potential function 
$f$ on a connected Riemannian manifold $(M, g)$, there exists a unique smooth function $\rho$ such that $\nabla f=\rho \boldsymbol{u}$, where $\nabla f$ is the gradient of the potential function $f$. Thus, this unique function $\rho$ connects the gradient $\nabla f$ of the potential function $f$ and the concircular vector vector field $u$ and therefore, we call $\rho$ the connecting function of the concircular vector field $u$. It is interesting to observe that connecting function $\rho$ is helpful in finding characterizations of the $n$-sphere $\mathbf{S}^{n}(c)$ as well as the Euclidean space $\mathbf{E}^{n}$ (cf. Theorems 2-5). Moreover, in the last section, we observe that the connecting function $\rho$ also influences topology of the Riemannian manifold (cf. Theorems 7 and 8).

\section{Preliminaries}

Let $(M, g)$ be an $n$-dimensional Riemannian manifold $(M, g)$ and $u$ be a non-trivial concircular vector field on $(M, g)$ with potential function $f$ (see, e.g., [11]). Then,

$$
\nabla_{X} \boldsymbol{u}=f X, \quad X \in \mathfrak{X}(M),
$$

and the curvature tensor field $R$ of the Riemannian manifold $(M, g)$ satisfies

$$
R(X, Y) \boldsymbol{u}=X(f) Y-Y(f) X, \quad X, Y \in \mathfrak{X}(M),
$$

where

$$
R(X, Y) Z=\nabla_{X} \nabla_{Y} Z-\nabla_{Y} \nabla_{X} Z-\nabla_{[X, Y]} Z
$$

On an $n$-dimensional Riemannian manifold $(M, g)$, the Ricci tensor Ric is given by formula

$$
\operatorname{Ric}(X, Y)=\sum_{i=1}^{n} g\left(R\left(e_{i}, X\right) Y, e_{i}\right)
$$

where $\left\{e_{1}, . ., e_{n}\right\}$ is a local orthonormal frame on $M$. The Ricci operator $Q$ of the Riemannian manifold $(M, g)$ is a symmetric operator defined by

$$
g(Q X, Y)=\operatorname{Ric}(X, Y), \quad X, Y \in \mathfrak{H}(M) .
$$

The scalar curvature $S=\operatorname{Tr} Q$ of the Riemannian manifold is the trace of the Ricci operator $Q$. The gradient $\nabla S$ of the scalar curvature satisfies (cf. $[19,20]$ )

$$
\frac{1}{2} \nabla S=\sum_{i=1}^{n}(\nabla Q)\left(e_{i}, e_{i}\right),
$$

where the covariant derivative is defined by

$$
(\nabla Q)(X, Y)=\nabla_{X} Q Y-Q \nabla_{X} Y
$$

Thus, using Equations (2) and (3), we conclude

$$
\operatorname{Ric}(Y, \boldsymbol{u})=-(n-1) Y(f) .
$$

Hence, we have

$$
Q(\boldsymbol{u})=-(n-1) \nabla f,
$$

where $\nabla f$ is the gradient of the potential function $f$.

We also have a smooth function $h: M \rightarrow R$ on a Riemannian manifold $(M, g)$ associated to concircular vector field $u$ and defined by

$$
h=\frac{1}{2}\|u\|^{2} .
$$


Then, using Equation (1), we find the gradient $\nabla h$ of the smooth function $h$

$$
\nabla h=f u .
$$

Note that the Hessian operator $A_{\varphi}$ of a smooth function $\varphi: M \rightarrow R$ on a Riemannian manifold $(M, g)$, and its Laplacian $\Delta \varphi$ are defined by

$$
A_{\varphi} X=\nabla_{X} \nabla \varphi, \quad \Delta \varphi=\operatorname{div} \nabla \varphi=\operatorname{Tr} A_{\varphi},
$$

respectively, where

$$
\operatorname{div} X=\sum_{i=1}^{n} g\left(\nabla_{e_{i}} X, e_{i}\right) .
$$

The Hessian $\operatorname{Hess}(\varphi)$ is defined by

$$
\operatorname{Hess}(\varphi)(X, Y)=g\left(A_{\varphi} X, Y\right), \quad X, Y \in \mathfrak{X}(M) .
$$

Note that if $\varphi$ is a non-constant smooth function on a compact Riemannian manifold $(M, g)$ satisfying relation

$$
\int_{M} \varphi=0,
$$

then the minimal principle gives

$$
\int_{M}\|\nabla \varphi\|^{2} \geq \lambda_{1} \int_{M} \varphi^{2},
$$

where $\lambda_{1}$ is a first non-zero eigenvalue of the Laplace operator $\Delta$ acting on smooth functions of $M$.

Recall that the Laplace operator $\Delta$ acting on smooth vector fields on an $n$-dimensional Riemannian manifold $(M, g)$ is defined by

$$
\Delta X=\sum_{i=1}^{n}\left(\nabla_{e_{i}} \nabla_{e_{i}} X-\nabla_{\nabla_{e_{i}} e_{i}} X\right), \quad X \in \mathfrak{X}(M),
$$

where $\left\{e_{1}, \ldots, e_{n}\right\}$ is an orthonormal frame on $M$. A smooth vector field $X$ is said to be harmonic if $\Delta X=0$.

\section{Connecting Functions of Concircular Vector Fields}

In this section, first we show that for a non-trivial concircular vector field $u$ with potential function $f$ on a connected Riemannian manifold $(M, g)$, there exists a unique smooth function $\rho: M \rightarrow R$, which we call the connecting function of the concircular vector field $u$. Then, it is shown that the connecting function $\rho$ can be used to find characterizations of the $n$-sphere $\mathbf{S}^{n}(c)$ as well as the Euclidean space $\mathbf{E}^{n}$.

Theorem 1. Let $\boldsymbol{u}$ be a non-trivial concircular vector field with potential function $f$ on a connected Riemannian manifold $(M, g)$. Then, there exists a unique function $\rho: M \rightarrow R$ satisfying

$$
\nabla f=\rho \boldsymbol{u} .
$$


Proof. Let $u$ be a non-trivial concircular vector field with potential function $f$ on a connected Riemannian manifold $(M, g)$. Then, for the smooth function $h=\frac{1}{2}\|\boldsymbol{u}\|^{2}$, using Equations (5) and (6), we find the following expression for the Hessian operator $A_{h}$ :

$$
A_{h}(X)=X(f) \boldsymbol{u}+f^{2} X
$$

Thus, the Hessian Hess $(h)$ of the smooth function $h$ is given by

$$
\operatorname{Hess}(h)(X, Y)=X(f) g(\boldsymbol{u}, Y)+f^{2} g(X, Y), \quad X, Y \in \mathfrak{X}(M) .
$$

Now, as the Hess $(h)$ is symmetric, Equation (7) implies

$$
X(f) g(\boldsymbol{u}, Y)=Y(f) g(\boldsymbol{u}, X)
$$

and through which, we conclude

$$
X(f) \boldsymbol{u}=g(\boldsymbol{u}, X) \nabla f .
$$

Replacing $X$ by $u$ in above equation, we get

$$
\boldsymbol{u}(f) u=\|\boldsymbol{u}\|^{2} \nabla f
$$

which on taking the inner product with $\nabla f$, gives

$$
\|\boldsymbol{u}\|^{2}\|\nabla f\|^{2}=\boldsymbol{u}(f)^{2}=g(\boldsymbol{u}, \nabla f)^{2} .
$$

Above equation confirms that vector fields $u$ and $\nabla f$ are parallel. Hence, there exists a smooth function $\rho: M \rightarrow R$ such that

$$
\nabla f=\rho \boldsymbol{u}
$$

If there is another function $\sigma: M \rightarrow R$, satisfying $\nabla f=\sigma \boldsymbol{u}$, then we have $(\rho-\sigma) \boldsymbol{u}=0$, which on connected $M$ implies that either $\rho=\sigma$ or $\boldsymbol{u}=0$. However, $\boldsymbol{u}=0$ in Equation (1), gives $f=0$, a contradiction to the fact that $u$ is a non-trivial concircular vector field. Hence, $\rho=\sigma$, that is $\rho$ is a unique function satisfying Equation (8).

The unique function $\rho$ guaranteed by Theorem 1 that is associated to the non-trivial concircular vector field $\boldsymbol{u}$ with potential function $f$ on a connected Riemannian manifold $(M, g)$ connects the vector field $\nabla f$ to the vector field $u$. Therefore, we call the function $\rho$ the connecting function of the non-trivial concircular vector field $u$. In the following results, we show that the connecting function $\rho$ can be used to characterize a $n$-sphere $\mathbf{S}^{n}(c)$ of constant curvature $c$. In Theorems $2-4$, we observe that a Riemannian manifold $(M, g)$ admitting a concircular vector field with connecting function $\rho$ is isometric to the $n$-sphere $S^{n}(c)$, then the connecting function $\rho=-c$ holds.

Theorem 2. An n-dimensional compact and connected Riemannian manifold $(M, g)$ admits a non-trivial concircular vector field $\boldsymbol{u}$ with potential function $f$ such that the connecting function $\rho$ is a constant along the integral curves of $\boldsymbol{u}$, if and only if $(M, g)$ is isometric to the $n$-sphere $\mathbf{S}^{n}(c)$.

Proof. Suppose $(M, g)$ is an $n$-dimensional compact and connected Riemannian manifold admits a non-trivial concircular vector field $u$ with potential function $f$ such that the connecting function $\rho$ is a constant along the integral curves of $\boldsymbol{u}$, that is, $\boldsymbol{u}(\rho)=0$. Then, using

$$
\nabla f=\rho \boldsymbol{u},
$$


the Hessian operator $A_{f}$ of the potential function $f$ is computed by taking covariate derivative in above equation, which is given by

$$
A_{f} X=X(\rho) \boldsymbol{u}+\rho f X, \quad X \in \mathfrak{X}(M) .
$$

Note that, using Equation (7), we conclude divu $=n f$, and integrating this equation leads to

$$
\int_{M} f=0 .
$$

If $f$ is a constant, then above equation concludes that $f=0$, which is contrary to the assumption that $u$ is a non-trivial circular vector field. Hence, the potential function $f$ is a non-constant function. Now, using the symmetry of the Hessian operator $A_{f}$ in Equation (9), we conclude that

$$
X(\rho) g(\boldsymbol{u}, Y)=Y(\rho) g(\boldsymbol{u}, X),
$$

and it implies that

$$
X(\rho) \boldsymbol{u}=g(\boldsymbol{u}, X) \nabla \rho .
$$

Replacing $X$ by $\boldsymbol{u}$ in the above equation and using $\boldsymbol{u}(\rho)=0$, we conclude

$$
\|\boldsymbol{u}\|^{2} \nabla \rho=0 .
$$

However, $u \neq 0$ being a non-trivial concircular vector field, the above equation on connected $M$ gives $\nabla \rho=0$, that is, the connecting function $\rho$ is a constant. Moreover, the constant $\rho$ has to be a non-zero constant. If $\rho=0$, then Theorem 1 implies $f$ is a constant, which is ruled out in the previous paragraph. Taking trace in Equation (9), we get $\Delta f=n \rho f$, that is, the non-constant function $f$ is eigenfunction of the Laplace operator $\Delta$ acting on smooth functions on $M$. Since $M$ is compact, we conclude $n \rho<0$, that is, the non-zero constant $\rho<0$. We put $\rho=-c, c>0$ and we have

$$
\nabla f=-c u
$$

Taking covariant derivative in Equation (10) with respect to $X \in \mathfrak{X}(M)$ and using Equation (1), we get

$$
\nabla_{X} \nabla f=-c f X, \quad X \in \mathfrak{X}(M) .
$$

Hence, the non-constant function $f$ satisfies Obata's differential equation (Equation (11)) (cf. [13]) and, thus, the Riemannian manifold $(M, g)$ is isometric to the sphere $\mathbf{S}^{n}(c)$.

Conversely, we know that $\mathbf{S}^{n}(c)$ is a hypersurface of the Euclidean space $\mathbf{E}^{n+1}$ with unit normal $N$ and the Weingarten map $A=-\sqrt{c} I$. We take a non-zero constant vector field $Z$ on the Euclidean space $\mathbf{E}^{n+1}$, whose restriction to $\mathbf{S}^{n}(c)$, can be expressed as $Z=\boldsymbol{u}+s N$, where $\boldsymbol{u}$ is the tangential component of $Z$ and $s$ is a smooth function $s=\langle Z, N\rangle$ on the sphere $\mathbf{S}^{n}(c)$, and $\langle$,$\rangle is the Euclidean metric on \mathbf{E}^{n+1}$. Taking $X \in \mathfrak{X}\left(\mathbf{S}^{n}(c)\right)$, we get $X(s)=\langle Z, \sqrt{c} X\rangle=\sqrt{c} g(\boldsymbol{u}, X)$, where $g$ is the induced metric on $\mathbf{S}^{n}(c)$. Thus, we conclude

$$
\nabla s=\sqrt{c} \boldsymbol{u}
$$

Now, as $Z$ is a constant vector field, using the Euclidean connection $D$ on the Euclidean space $\mathbf{E}^{n+1}$, we have $D_{X} Z=0$. For $X, Y \in \mathfrak{X}\left(\mathbf{S}^{n}(c)\right.$, using the Gauss formula for hypersurface $D_{X} Y=$ $\nabla_{X} Y-\sqrt{c} g(X, Y) N$, we compute

$$
\begin{aligned}
0 & =\left\langle D_{X} Z, Y\right\rangle=X\langle Z, Y\rangle-\left\langle Z, \nabla_{X} Y-\sqrt{c} g(X, Y) N\right\rangle \\
& =X g(\boldsymbol{u}, Y)-g\left(\boldsymbol{u}, \nabla_{X} Y\right)+\sqrt{c} \operatorname{sg}(X, Y) \\
& =g\left(\nabla_{X} \boldsymbol{u}, Y\right)+\sqrt{c s} g(X, Y),
\end{aligned}
$$


and conclude

$$
\nabla_{X} \boldsymbol{u}=-\sqrt{c s} X, \quad X \in \mathfrak{X}\left(\mathbf{S}^{n}(c)\right) .
$$

Hence, $u$ is concircular vector field on $\mathbf{S}^{n}(c)$, with potential function $f=-\sqrt{c s}$, which gives $\nabla f=-\sqrt{c} \nabla s$. Using Equation (12), we get

$$
\nabla f=-c u
$$

Suppose $f=0$, which implies $s=0$ and, in view of Equation (12), $u=0$, that is $Z=0$ on $\mathbf{S}^{n}(c)$. As $Z$ is a constant vector field, we get $Z=0$ on $\mathbf{E}^{n+1}$, which gives a contradiction to the fact that $Z$ is a non-zero constant vector field. Hence, $f \neq 0$, that is, $u$ is a non-trivial concircular vector field with potential function $f$. Then, Equation (14) implies that the connecting function $\rho=-c$, which is a constant.

Theorem 3. An n-dimensional complete and simply connected Riemannian manifold $(M, g)$ admits a non-trivial concircular vector field $\boldsymbol{u}$ with potential function $f$ such that $\Delta \boldsymbol{u}=-\lambda \boldsymbol{u}$ for a constant $\lambda>0$, if and only if $(M, g)$ is isometric to the $n$-sphere $\mathbf{S}^{n}(\lambda)$.

Proof. Suppose $\boldsymbol{u}$ is a non-trivial concircular vector field on $(M, g)$ with potential function $f$ and connecting function $\rho$ such that $\Delta \boldsymbol{u}=-\lambda \boldsymbol{u}, \lambda>0$. Using Equation (1) and a local orthonormal frame $\left\{e_{1}, \ldots, e_{n}\right\}$ on $M$, by a straight forward computation, we get $\Delta \boldsymbol{u}=\nabla f$. Thus, Theorem 1 gives $-\lambda \boldsymbol{u}=\rho \boldsymbol{u}$, that is, $(\rho+\lambda) \boldsymbol{u}=0$. Since a simply connected $M$ is also connected and $\boldsymbol{u}$ being a non-trivial concircular vector field $u \neq 0$, we must have $\rho=-\lambda$ and, consequently, Theorem 1 implies $\nabla f=-\lambda \boldsymbol{u}$, which, on using Equation (1), gives

$$
\nabla_{X} \nabla f=-\lambda f X, \quad X \in \mathfrak{X}(M) .
$$

If potential function $f$ is a constant, then the above equation implies $f=0$ (as $\lambda>0$ ), which is contrary to the assumption that $f$ is potential function of the non-trivial concircular vector field $\boldsymbol{u}$. Hence, Equation (15) is Obata's differential equation for non-constant function $f$ and positive constant $\lambda$, which proves that $(M, g)$ is isometric to $\mathbf{S}^{n}(\lambda)$.

Conversely, on $\mathbf{S}^{n}(c)$, as in the proof of Theorem 2, there is a non-trivial concircular vector field $u$ with potential function $f$ and connecting function $\rho$ that satisfy Equations (12)-(14), which implies $\Delta \boldsymbol{u}=-c \boldsymbol{u}$.

Theorem 4. An n-dimensional complete and simply connected Riemannian manifold $(M, g)$ admits a non-trivial concircular vector field $\boldsymbol{u}$ with potential function $f$ and connecting function $\rho$ satisfying $(i) g(\nabla f, \nabla \rho)=0$ and (ii) $\operatorname{Ric}(\nabla f, \nabla f)>0$, if and only if $(M, g)$ is isometric to the $n$-sphere $\mathbf{S}^{n}(c)$.

Proof. Suppose $u$ is a non-trivial concircular vector field with potential function $f$ and connecting function $\rho$ on an $n$-dimensional Riemannian manifold $(M, g)$ satisfying

$$
g(\nabla f, \nabla \rho)=0 \quad \text { and } \quad \operatorname{Ric}(\nabla f, \nabla f)>0 .
$$

Then, using Theorem 1, in the above equations, we conclude

$$
\rho \boldsymbol{u}(\rho)=0 \quad \text { and } \quad \rho^{2} \operatorname{Ric}(\boldsymbol{u}, \boldsymbol{u})>0,
$$

that is, $\boldsymbol{u}(\rho)=0$. Using the symmetry of Hessian operator in Equation (9), we have

$$
X(\rho) g(\boldsymbol{u}, Y)=Y(\rho) g(\boldsymbol{u}, X), \quad X, Y \in \mathfrak{X}(M),
$$


and taking $X=\boldsymbol{u}$ in the above equation yields $Y(\rho)\|\boldsymbol{u}\|^{2}=0, Y \in \mathfrak{X}(M)$. As $u$ is a non-trivial concircular vector field, we must have $Y(\rho)=0, Y \in \mathfrak{X}(M)$, that is, $\rho$ is a constant and in view of second equation in Equation (16), constant $\rho \neq 0$. Now, Equation (4) and Theorem 1 imply

$$
\operatorname{Ric}(\boldsymbol{u}, \boldsymbol{u})=-(n-1) \rho\|\boldsymbol{u}\|^{2} .
$$

Combining Equations (16) and (17), we conclude that the non-zero constant $\rho<0$. Taking $\rho=-c$, $c>0$, Theorem 1 gives $\nabla f=-c u$, where $f$ has to be non-constant, otherwise we would have $\boldsymbol{u}=0$, which is ruled out. Hence, using Equation (1), we get the Obata's differential equation

$$
\nabla_{X} \nabla f=-c f X, \quad X \in \mathfrak{X}(M),
$$

proving that $(M, g)$ is isometric to $\mathbf{S}^{n}(c)$.

The converse trivially follows through the proof of Theorem 2.

\section{Characterizations of Euclidean Spaces}

In this section, we are interested in finding characterizations of a Euclidean space using non-trivial concircular vector fields.

Theorem 5. An n-dimensional complete and connected Riemannian manifold $(M, g)$ admits a non-trivial concircular vector field $\boldsymbol{u}$ with potential function $f$ satisfying $\operatorname{Ric}(\nabla f, \nabla f)=0$, if and only if $(M, g)$ is isometric to the Euclidean space $\mathbf{E}^{n}$.

Proof. Suppose $(M, g)$ is an $n$-dimensional complete and connected Riemannian manifold $(M, g)$ that admits a non-trivial concircular vector field $u$ with potential function $f$, connecting function $\rho$ and the Ricci curvature satisfies

$$
\operatorname{Ric}(\nabla f, \nabla f)=0 .
$$

Using Equation (4) and Theorem 1, we have

$$
Q(\nabla f)=-(n-1) \rho \nabla f,
$$

which, in view of Equation (18), gives

$$
\text { - }(n-1) \rho\|\nabla f\|^{2}=0 .
$$

Note that, if $\rho=0$, then Theorem 1 gives $\nabla f=0$, that is, $f$ is a constant. Thus, as $M$ is connected, Equation (19), in both its outcomes, implies that $f$ is a constant. Now, observe that the constant $f \neq 0$, owing to the fact that $u$ is non-trivial. Using Equation (5), for the function $h=\frac{1}{2}\|u\|^{2}$, we find the following expression for its Hessian operator

$$
A_{h} X=f^{2} X,
$$

and, consequently, we have

$$
\operatorname{Hess}(h)=c g,
$$

where $c=f^{2}$ is a non-zero constant. Notice through Equation (5) that the function $h$ is not a constant, for, if $h$ were to be a constant, as $f \neq 0$, it would imply $u=0$, a contradiction. Hence, the non-constant function $h$ satisfies Equation (20) for a non-zero constant $c$, proving that the complete and connected Riemannian manifold $(M, g)$ is isometric to the Euclidean space $\mathbf{E}^{n}$ (cf. Theorem 1, [15]). 
Conversely, consider the position vector field

$$
\boldsymbol{u}=\sum_{i=1}^{n} x^{i} \frac{\partial}{\partial x^{i}}
$$

on the Euclidean space $\mathbf{E}^{n}$, where $x^{1}, \ldots, x^{n}$ are Euclidean coordinates, which satisfies $\nabla_{X} \boldsymbol{u}=X$, $X \in \mathfrak{X}\left(\mathbf{E}^{n}\right)$, where $\nabla$ is the Euclidean connection on $\mathbf{E}^{n}$. Thus, $u$ is a non-trivial concircular vector field on $\mathbf{E}^{n}$ with potential function $f=1$ and connecting function $\rho=0$, which satisfies the condition in the statement of the theorem.

Our next result shows that harmonic concircular vector fields characterize Euclidean spaces.

Theorem 6. An n-dimensional complete and connected Riemannian manifold $(M, g)$ admits a non-trivial concircular vector field $\boldsymbol{u}$ that satisfies $\Delta \boldsymbol{u}=0$, if and only if $(M, g)$ is isometric to the Euclidean space $\mathbf{E}^{n}$.

Proof. Suppose $u$ is a non-trivial concircular vector field with potential function $f$ on an $n$-dimensional complete and connected Riemannian manifold $(M, g)$, which satisfies $\Delta u=0$. Using Equation (1), we compute

$$
\Delta \boldsymbol{u}=\nabla f
$$

Hence, the potential function $f$ is a constant and this constant $f \neq 0$ as $u$ is a non-trivial concircular vector field. Now, Equation (5), with $f$ a constant gives

$$
\operatorname{Hess}(h)=c g,
$$

where $c=f^{2}$ is a non-zero constant. Hence, $(M, g)$ is isometric to the Euclidean space $\mathbf{E}^{n}$.

The converse is trivial, as the position vector field $u$ on the Euclidean space $\mathbf{E}^{n}$ is harmonic.

\section{Influence of Concircular Vector Fields on Topology}

In this section, we observe that because of the connecting function, we can exhibit the influence of non-trivial concircular vector fields on topology of the Reimannian manifolds. Our observations depend on already known results and therefore results in this sections are simply trivial applications of known results in differential topology. Recall that, by Reeb's theorem, if a compact smooth manifold $M$ admits a smooth function $F: M \rightarrow R$ with exactly two critical points which are non-degenerate, then $M$ is homeomorphic to an $n$-sphere $S^{n}$. Moreover, it was later observed by Milnor (cf. Theorem 1 , p. 166, [21]) that this result holds even if the two critical points are degenerate. Using this modified Reeb's theorem, we have the following trivial consequence:

Theorem 7. If an $n$-dimensional compact and connected Riemannian manifold $(M, g)$ admits a non-trivial concircular vector field $\boldsymbol{u}$ with potential function $f$ and connecting function $\rho$ such that $\rho(p) \neq 0$ for each $p \in M$ and vector field $\boldsymbol{u}$ has only two zeros, then $M$ is homeomorphic to an $n$-sphere.

Proof. Using Theorem 2, we have $\nabla f=\rho \boldsymbol{u}$, and the vector field $\boldsymbol{u}$ has two zeros say at $p$ and $q \in M$. Then, as connecting function $\rho(x) \neq 0$ on $M$, points $p, q$ are critical points of the potential function $f$. Thus, the smooth function $f$ has exactly two critical points, which proves that $M$ is homeomorphic to $n$-sphere.

Consider a non-trivial concircular vector field $u$ that is nowhere zero on an $n$-dimensional connected Riemannian manifold $(M, g)$ with potential function $f$ and connecting function $\rho(p) \neq 0$, 
$p \in M$. Then, by Theorem 1, the potential function $f$ has no critical points. If we define a smooth vector field $\xi$ on $M$ by

$$
\xi=\frac{\nabla f}{\|\nabla f\|^{2}}
$$

then, as $\xi(f)=1$, the local flow $\left\{\phi_{t}\right\}$ of $\xi$ satisfies

$$
f\left(\phi_{t}(p)\right)=f(p)+t
$$

which on using escape lemma (cf. [22]) proves that $\xi$ is a complete vector field and $\left\{\phi_{t}\right\}$ is the global flow. Moreover, observe that $f: M \rightarrow R$ is a submersion, consequently, the lever set $M_{p}=f^{-1}\{f(p)\}$ is a compact hypersurface of $M$. Now, we have the following:

Theorem 8. If an n-dimensional connected Riemannian manifold $(M, g)$ admits a non-trivial concircular vector field $\boldsymbol{u}, \boldsymbol{u}(p) \neq 0, p \in M$, with potential function $f$ and connecting function $\rho$ such that $\rho(p) \neq 0$ for each $p \in M$, then $M$ is diffeomorphic to $N \times R$ for some compact smooth manifold $N$.

Proof. For $p \in M$, we denote by $M_{p}$ the level set $f^{-1}\{f(p)\}$ of $f$, which is a compact hypersurface of $M$. We define $F: M_{p} \times R \rightarrow M$ by

$$
F(q, t)=\phi_{t}(q),
$$

which is a smooth map. First, we show that $F$ is a surjective: Take $x \in M$; then, we can find $s \in R$, such that $\phi_{s}(x)=m \in M_{p}$, with $x=\phi_{-s}(m)$. Consequently,

$$
F(m,-s)=x
$$

Next, we show that $F$ is an injective: Take $\left(q_{1}, t_{1}\right),\left(q_{2}, t_{2}\right) \in M_{p} \times R$ such that $F\left(q_{1}, t_{1}\right)=F\left(q_{2}, t_{2}\right)$. Then, we have $\phi_{t_{1}}\left(q_{1}\right)=\phi_{t_{2}}\left(q_{2}\right)$ and, using Equation (21), we get

$$
f\left(q_{1}\right)+t_{1}=f\left(q_{2}\right)+t_{2} .
$$

However, as $q_{1}, q_{2} \in M_{p}$, we have $f\left(q_{1}\right)=f\left(q_{2}\right)$. Thus, we get $t_{1}=t_{2}$. and $\phi_{t_{1}}\left(q_{1}\right)=\phi_{t_{1}}\left(q_{2}\right)$ implies $q_{1}=q_{2}$. Hence, $F$ is an injective. Finally, we have

$$
F^{-1}(x)=(m,-s)=\left(\phi_{s}(x),-s\right)
$$

is also smooth. Hence, $F$ is a diffeomorphism.

Author Contributions: Conceptualization and methodology, S.D. and I.A.-D.; formal analysis, O.B.; writing original draft preparation, S.D. and I.A.-D.; writing - review and editing, S.D. and O.B.; supervision, S.D. and O.B.; project administration, I.A.-D.; and funding acquisition, I.A.-D. All authors have read and agreed to the published version of the manuscript.

Funding: This research was supported by King Saud University, Deanship of Scientific Research, College of Science Research Centre.

Conflicts of Interest: The authors declare no conflicts of interest.

\section{References}

1. Chen, B.-Y. Some results on concircular vector fields and their applications to Ricci solitons. Bull. Korean Math. Soc. 2015, 52, 1535-1547. [CrossRef]

2. Chen, B.-Y.; Deshmukh, S.; Ishan, A.A. On Jacobi-type vector fields on Riemannian manifolds. Mathematics 2019, 7, 1139. [CrossRef] 
3. Chen, B.-Y.; Deshmukh, S. Some results about concircular vector fields on Riemannian manifolds. Filomat 2020, 34. Available online: http://journal.pmf.ni.ac.rs/filomat/index.php/filomat/article/view/12274 (accessed on 24 February 2020).

4. Deshmukh, S. Conformal Vector Fields and Eigenvectors of Laplacian Operator. Math. Phys. Anal. Geom. 2012, 15, 163-172. [CrossRef]

5. Deshmukh, S.; Al-Solamy, F. Conformal gradient vector fields on a compact Riemannian manifold. Colloq. Math. 2008, 112, 157-161. [CrossRef]

6. Deshmukh, S. Jacobi-type vector fields and Ricci soliton. Bull. Math. Soc. Sci. Math. Roumanie 2012, 55, 41-50.

7. Deshmukh, S.; Khan, V.A. Geodesic vector fields and Eikonal equation on a Riemannian manifold. Indag. Math. 2019, 30, 542-552. [CrossRef]

8. Deshmukh, S.; Turki, N. A note on $\varphi$-analytic conformal vector fields. Anal. Math. Phys. 2019, 9, $181-195$. [CrossRef]

9. Deshmukh, S. Characterizing spheres and Euclidean spaces by conformal vector field. Annali di Matematica Pura ed Applicata 2017, 196, 2135-2145. [CrossRef]

10. Deshmukh, S.; Peška, P.; Bin Turki, N. Geodesic Vector Fields on a Riemannian Manifold. Mathematics 2020, 8, 137. [CrossRef]

11. Fialkow, A. Conformal geodesics. Trans. Amer. Math. Soc. 1939 45, 443-473. [CrossRef]

12. Ishihara, S. On infinitesimal concircular transformations. Kodai Math. Sem. Rep. 1960, 12, 45-56. [CrossRef]

13. Obata, M. Conformal transformations of Riemannian manifolds. J. Diff. Geom. 1970, 4, 311-333. [CrossRef]

14. Obata, M. The conjectures about conformal transformations. J. Diff. Geom. 1971, 6, 247-258. [CrossRef]

15. Pigola, S.; Rimoldi, M.; Setti, A.G. Remarks on non-compact gradient Ricci solitons. Math. Z. 2011, 268, 777-790. [CrossRef]

16. Yano, K. Concircular geometry I. Concircular transformations. Proc. Imp. Acad. Tokyo 1940, 16, 195-200. [CrossRef]

17. Shandra, I.G. Concircular vector fields on semi-Riemannian spases. J. Math. Sci. 2007, 142, $2419-2435$. [CrossRef]

18. Takeno, H. Concircular scalar field in spherically symmetric space-times I. Tensor 1967, 20, 167-176.

19. Besse, A.L. Einstein Manifolds; Springer: Berlin/Heidelberg, Germany, 1987.

20. Chen, B.-Y. Concircular vector fields and pseudo-Kaehler manifolds. Kragujevak J. Math. 2016, 40, 7-14. [CrossRef]

21. Milnor, J. Differenial Topology. In Lectures on Modern Mathematics III; Saaty, T.L., Ed.; John Wiley \& Sons: Hoboken, NJ, USA, 1964.

22. Lee, J.M. Introduction to Smooth Manifolds; GMT-218; Springer: Berlin/Heidelberg, Germany, 2003. 